International Journal of Pure and Applied Mathematics

Volume 82 No. 4 2013, 615-621

ISSN: 1311-8080 (printed version); ISSN: 1314-3395 (on-line version)

url: http://www.ijpam.eu

doi: http://dx.doi.org/10.12732/ijpam.v82i4.10

ijpam.eu

\title{
ON CONVOLUTION OF SOME TYPE OF THE NUMBERS CONNECTED WITH GENERALIZED REPUNITS
}

\author{
Pavel Trojovský \\ University of Hradec Králové \\ Faculty of Science \\ Department of Mathematics \\ 62, Rokitanského, 50003, Hradec Králové, CZECH REPUBLIC
}

Abstract: We will concentrate on special types of numbers

$$
J_{n}(k)=\sum_{i=0}^{n-2}\left(\begin{array}{c}
n \\
i
\end{array}\right) k^{n-2-i},
$$

where $k$ is any nonnegative integer and $n$ is any positive integer greater than 1 . These numbers are a generalization of generalized repunits $R_{n}(b)$. In this paper some results about divisibility of $J_{n}(k)$ are stated. Further the generating function and a $m$-fold convolution formula for the numbers $J_{n}(k)$ is found.

AMS Subject Classification: 11A51, 11A07, $11 \mathrm{Y} 11$

Key Words: recurrences, repunits, congruences, generating functions, convolution

\section{Introduction}

The term repunit was coined by Beiler [2] in 1966. A repunit $R_{n}$ is any integer written in decimal form as a string of 1 's. The numbers 1, 11, 111, 1111, 11111, etc., are examples of repunits. Thus repunits have the form $R_{n}=\frac{10^{n}-1}{9}$. The great effort was devoted to searching of repunit primes, thus such primes which are any repunits and they are also prime numbers, see Reuschle [11], Hoppe [7], Lehmer [10] and Kraitchik [9], Williams and Dubner [16]. In recent time

Received: September 28, 2012

(c) 2013 Academic Publications, Ltd. url: www.acadpubl.eu 
four probably prime repunits $R_{49081}, R_{86453}, R_{109297}, R_{270343}$ have known (see Dubner [4] and [5], Baxter [1] and Voznyy and Budnyy [14]). For more facts about repunits see Yates [17]. Snyder [12] extended the notation repunit to one in which for some integer $b \geq 2$ by this way

$$
R_{n}(b)=\frac{b^{n}-1}{b-1}
$$

They are called as generalized repunits or repunits to base $b$ and consist of a string of 1's when written in base $b$. Some facts on the divisibility and primality of $R_{n}(b)$ can be found in Williams [15], Jaroma [8] and Dubner [3]. We have found $m$-fold convolution formula for $R_{n}(b)$ and some their divisibility properties in [13]. In this paper we will investigate a generalization of generalized repunits $R_{n}(k+1)$, which are created by subtracting the linear term in $(k+1)^{n}$ and dividing by the trivial divisor $k^{2}$

$$
J_{n}(k)=\frac{(k+1)^{n}-n k-1}{k^{2}},
$$

It is easy to realize that all the numbers $J_{n}(k)$ are nonnegative integers for arbitrary positive integers $k$ and $n$. Hence we can also write the numbers $J_{n}(k)$ in the form

$$
J_{n}(k)=\sum_{i=0}^{n-2}\left(\begin{array}{l}
n \\
i
\end{array}\right) k^{n-2-i}
$$

with $J_{0}(k)=J_{1}(k)=0$.

\section{The Main Results}

The main results established in this paper concern some congruences for the numbers $J_{n}(k)$ and a $m$-fold convolution formula for $J_{n}(k)$ is derived. They are expressed in the following theorems.

Theorem 1. Let $a, b, l$ be any positive integers. Then

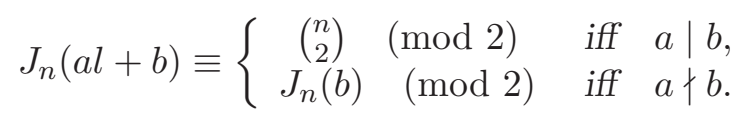

holds for $n \geqq 1$.

Corollary 2. Let $a, m, l$ be any positive integers. Then

$$
J_{a m}(a l) \equiv\left\{\begin{array}{ccccc}
0 & (\bmod a), & \multicolumn{1}{c}{a \equiv 1 \quad(\bmod 2)} & \vee \\
& & (a \equiv 0 \quad(\bmod 2) & \wedge m \equiv 0 & (\bmod 2)) \\
\frac{a}{2} & (\bmod a), & a \equiv 0 & (\bmod 2) \wedge m \equiv 1 & (\bmod 2)
\end{array}\right.
$$


Theorem 3. Let $k, m \geq 2, n$ be any positive integers. Then the following formula for the numbers $J_{n}(k)$ holds

$$
\begin{aligned}
\sum_{\substack{n_{1}, n_{2}, \ldots n_{m} \\
n_{1}+n_{2}+\cdots+n_{m}=n}} J_{n_{1}}(k) J_{n_{2}}(k) \cdots J_{n_{m}}(k)= \\
\quad=\sum_{l=0}^{n-2 m}\left(\begin{array}{c}
l+2 m-1 \\
2 m-1
\end{array}\right)\left(\begin{array}{c}
n-m-l-1 \\
m-1
\end{array}\right)(k+1)^{n-2 m-l} .
\end{aligned}
$$

\section{Some Lemmas and Preliminary Results}

(i) Recurrence relations With respect to $(2)$ the numbers $J_{n}(k)$ satisfy a nonhomogeneous linear difference equation of the second order and the roots of the characteristic equation must be $\lambda_{1}=k+1$ and $\lambda_{2}=1$. Hence the recurrence for the numbers $J_{n}(k)$ has the form

$$
J_{n+2}(k)-(k+2) J_{n+1}(k)+(k+1) J_{n}(k)=0
$$

and we obtain the sought after recurrence for the numbers $J_{n}(k)$ by putting (2) into the left side of (5) by the following way

$$
\begin{aligned}
J_{n+2}(k) & -(k+2) J_{n+1}(k)+(k+1) J_{n}(k) \\
& =\frac{1}{k^{2}}\left((k+1)^{n+2}-(n+2) k-1\right)-(k+2)(k+1)^{n+1} \\
& -(n+1) k-1+(k+1)\left((k+1)^{n}-n k-1\right)=1 .
\end{aligned}
$$

Therefore the numbers $J_{n}(k)$ satisfy the recurrence

$$
J_{n+2}(k)-(k+2) J_{n+1}(k)+(k+1) J_{n}(k)=1,
$$

with the initial conditions $J_{0}(k)=J_{1}(k)=0$. By an analogous procedure we can find for example recurrence

$$
J_{n+1}(k)-(k+1) J_{n}(k)=n, J_{0}(k)=0 .
$$


(ii) The generating function for the numbers $J_{n}(k)$. After arrangements we have for the generating function $j(x)$ of $J_{n}(k)$

$$
\begin{aligned}
j(x) & =\sum_{n=0}^{\infty} \frac{(k+1)^{n}-k n-1}{k^{2}} x^{n} \\
& =\frac{1}{k^{2}} \sum_{n=0}^{\infty}(k+1)^{n} x^{n}-\frac{1}{k} \sum_{n=0}^{\infty} n x^{n}-\frac{1}{k^{2}} \sum_{n=0}^{\infty} x^{n} \\
& =\frac{x^{2}}{(1-(k+1) x)(1-x)^{2}} .
\end{aligned}
$$

\section{The Proofs of the Main Theorems}

Proof of Theorem 1. We will consider the following two cases. Let $b \mid a$. Then

$$
\begin{aligned}
J_{n}(a l+b)=J_{n}(a m) & =\frac{(a m+1)^{n}-n(a m)-1}{(a m)^{2}}=\frac{\sum_{i=2}^{n}\left(\begin{array}{l}
n \\
i
\end{array}\right)(a m)^{i}}{(a m)^{2}} \\
& =\left(\begin{array}{c}
n \\
2
\end{array}\right)+\sum_{i=3}^{n}\left(\begin{array}{c}
n \\
i
\end{array}\right)(a m)^{i-2} \equiv\left(\begin{array}{c}
n \\
2
\end{array}\right) \quad(\bmod a) .
\end{aligned}
$$

Let $b \nmid a$. Then $a l+b \equiv b(\bmod a)$ and we have

$$
\begin{aligned}
J_{n}(a l+b) & =\frac{(a l+b+1)^{n}-n(a l+b)-1}{(a l+b)^{2}} \\
& =\frac{\left(a\left(\sum_{i=0}^{n-1}\left(\begin{array}{c}
n \\
i
\end{array}\right) a^{n-i-1} l^{n-i}(b+1)^{i}-n l\right)+(b+1)^{n}-n b-1\right)}{(a l+b)^{2}} \\
& \equiv \frac{(b+1)^{n}-n b-1}{b^{2}}(\bmod a) .
\end{aligned}
$$

Proof of Corollary 2. As the congruence $x y \equiv y(\bmod 2 y)$ clearly holds for any odd integer $x$ and any integer $y$ congruence (4) implies the assertion. 
Proof of Theorem 6. We use the following well-known fact on the generating function. If any sequence $\left\langle a_{n}\right\rangle$ has the generating function $A(x)$ (for example see [6], p. 355) then the $m$-fold convolution of the sequence $\left\langle a_{n}\right\rangle$ with itself has $n$th term equal to

$$
\sum_{\substack{n_{1}, n_{2}, \ldots n_{m} \\ n_{1}+n_{2}+\cdots+n_{m}=n}} a_{n_{1}} a_{n_{2}} \cdots a_{n_{m}}
$$

and its generating function is $A^{m}(x)$. Thus, we have

$$
\begin{aligned}
\sum_{n=0}^{\infty} & \left(\sum_{\begin{array}{c}
n_{1}, n_{2}, \ldots n_{m} \\
n_{1}+n_{2}+\cdots+n_{m}=n
\end{array}} J_{n_{1}}(k) J_{n_{2}}(k) \cdots J_{n_{m}}(k)\right)^{n} \\
= & \left(\frac{x^{2}}{(1-x)^{2}(1-(k+1) x)}\right)^{m} .
\end{aligned}
$$

On the left side we get

$$
\begin{aligned}
& \frac{x^{2 m}}{(1-x)^{2 m}(1-(k+1) x)^{m}}=x^{2 m} \frac{1}{(1-x)^{2 m}} \frac{1}{(1-(k+1) x)^{m}} \\
& =x^{2 m}\left(\sum_{n=0}^{\infty}\left(\begin{array}{c}
n+2 m-1 \\
2 m-1
\end{array}\right) x^{n}\right)\left(\sum_{n=0}^{\infty}\left(\begin{array}{c}
n+m-1 \\
m-1
\end{array}\right)(k+1)^{n}\right) x^{n} \\
& =\sum_{n=0}^{\infty}\left(\sum_{l=0}^{n}\left(\begin{array}{c}
l+2 m-1 \\
2 m-1
\end{array}\right)\left(\begin{array}{c}
n-l+m-1 \\
m-1
\end{array}\right)(k+1)^{n-l}\right) x^{n+2 m} \\
& =\sum_{n=2 m}^{\infty} \sum_{l=0}^{n-2 m}\left(\begin{array}{c}
l+2 m-1 \\
2 m-1
\end{array}\right)\left(\begin{array}{c}
n-2 m-l+m-1 \\
m-1
\end{array}\right)(k+1)^{n-2 m-l} x^{n} \\
& =\sum_{n=0}^{\infty}\left(\sum_{l=0}^{n-2 m}\left(\begin{array}{c}
l+2 m-1 \\
2 m-1
\end{array}\right)\left(\begin{array}{c}
n-m-l-1 \\
m-1
\end{array}\right)(k+1)^{n-2 m-l}\right) x^{n} .
\end{aligned}
$$

Therefore

$$
\begin{aligned}
\sum_{\substack{n_{1}, n_{2}, \ldots, n_{m} \\
n_{1}+n_{2}+\cdots+n_{m}=n}} J_{n_{1}}(k) J_{n_{2}}(k) \cdots J_{n_{m}}(k)= \\
=\sum_{l=0}^{n-2 m}\left(\begin{array}{c}
l+2 m-1 \\
2 m-1
\end{array}\right)\left(\begin{array}{c}
n-m-l-1 \\
m-1
\end{array}\right)(k+1)^{n-2 m-l} .
\end{aligned}
$$




\section{Acknowledgments}

This work was supported by the specific research 2103 UHKCZ.

\section{References}

[1] L. Baxter, R86453 is a New Probable Prime Repunit (2000), http://tech.groups.yahoo.com/group/primeform/message/1132

[2] A.H. Beiler, Recreations in the Theory of Numbers: The Queen of Mathematics Entertains, New York, Dover (1966).

[3] H. Dubner, Generalized repunit primes, Math. Comput., 61 (1993), 927930, doi: 10.1090/S0025-5718-1993-1185243-9.

[4] H. Dubner, Repunit R49081 is a probable prime, Math. Comput., 71, No. 238 (2002), 833-835.

[5] H. Dubner, New Repunit R(109297) (2007), http://listserv.nodak.edu/cgibin/wa.exe.

[6] R.L. Graham, D.E. Knuth, O. Patashnik, Concrete Mathematics: A Foundation for Computer Science, Addison-Wesley Publishing Company (1994).

[7] O. Hoppe, Proof of the primality of $\mathrm{n}=\left(10^{\wedge} 19-1\right) / 9$, Proc. Lond. Math. Soc., Records of Meetings of Dec. 6, 1917 and Feb. 14, 1918.

[8] J. Jaroma, Factoring Generalized Repunits, Irish Math. Soc. Bulletin, 59 (2007), 29-35.

[9] M. Kraitchik, Theorie des Nombres, Tome II. Gauthiers-Villars, Paris, France (1926).

[10] D.H. Lehmer, On the number $\mathrm{n}=\left(10^{\wedge} 23-1\right) / 9$. Bull. Amer. Math. Soc., 35, No. 3 (1929), 349-350.

[11] C.G. Reuschle, Mathematische Abhandlung, Enthaltend: Neue Zahlen- theoretische Tabellen. Koniglichen, Gymnasium zu Stuttgart (1856).

[12] W.M. Snyder, Factoring repunits, Am. Math. Monthly, 89 (1982), 462-466. 
[13] P. Trojovský, On convolution of generalized repunits, IJPAM, 79, No. 3 (2012).

[14] M. Voznyy, A. Budnyy, New PRP Repunit R(270343) (2007), http://listserv.nodak.edu/cgi-bin/wa.exe.

[15] H.C. Williams, E. Seah, Some primes of the form $\left(a^{\wedge} n-1\right) /(a-1)$, Math. Comput., 33 (1979), 1337-1342.

[16] H.C. Williams, H. Dubner, The primality of R1031, Math. Comput., 47 (1986), 703-711.

[17] S. Yates, Repunits and Repetends, Star Publ. Co., Boynton Beach, FL (1982). 
